\title{
COMPARAÇÃO DE DIMENSÕES DE PARCELAS PARA ANÁLISE DE VEGETAÇÃO EM UM FRAGMENTO DE MATA ATLÂNTICA, ARACAJU, SE
}

\author{
Paulo Gabriel de Oliveira $\mathrm{Neto}^{1 *}$, Anabel Aparecida de Mello ${ }^{2}$, Dráuzio Correia Gama ${ }^{1}$, \\ Ikaro Daniel de Carvalho Barreto ${ }^{3}$, Robério Anastácio Ferreira ${ }^{2}$, Nelson Yoshihiro Nakajima ${ }^{4}$ \\ ${ }^{1 *}$ Universidade Federal de Sergipe, Curso de Engenharia Florestal, São Cristovão, Sergipe, Brasil - paulo.floresta@ hotmail.com; \\ drauziogama@hotmail.com \\ ${ }^{2}$ Universidade Federal de Sergipe, Departamento de Ciências Florestais, São Cristovão, Sergipe, Brasil - anabel@ufs.br, raf@ufs.br \\ ${ }^{3}$ Universidade Federal Rural de Pernambuco, Programa de Pós-Graduação em Biometria e Estatística Aplicada, Dois Irmãos, \\ Recife, Pernambuco, Brasil - daniel.carvalho.ib@gmail.com \\ ${ }^{4}$ Universidade Federal do Paraná, Departamento de Engenharia Florestal, Curitiba, Paraná, Brasil - nelson.nakajima@ufpr.br
}

Recebido para publicação: 16/09/2014 - Aceito para publicação: 18/08/2015

\begin{abstract}
Resumo
Em um fragmento florestal de Mata Atlântica, localizado na Área de Proteção Ambiental (APA) Morro do Urubu, foi realizado um estudo de análise de vegetação com o objetivo de comparar duas dimensões de parcelas retangulares do mesmo tamanho. Para a execução do estudo, a área foi dividida em 314 unidades amostrais de $20 \mathrm{~m}$ x $100 \mathrm{~m}$ e, posteriormente após foram sorteados aleatoriamente 15 pontos amostrais, sendo instaladas 15 parcelas de $20 \mathrm{~m} \times 50 \mathrm{~m}\left(1000 \mathrm{~m}^{2}\right)$ e 15 parcelas de $10 \mathrm{mx} 100 \mathrm{~m}$ $\left(1000 \mathrm{~m}^{2}\right)$, a partir de cada um dos pontos sorteados. Nas parcelas, foram medidas a circunferência à altura do peito (CAP $(\mathrm{cm})-1,30 \mathrm{~m}$ de altura) e a altura total $(\mathrm{m})$ dos indivíduos que apresentaram $\mathrm{CAP}>31,4 \mathrm{~cm}$, utilizando-se fita métrica e vara telescópica, respectivamente. Além disso, foi coletado material botânico dos indivíduos para identificação científica das espécies. O teste estatístico utilizado para detectar se ocorreu diferença entre tratamentos independentes (diferentes dimensões das parcelas) foi o teste de Mann-Whitney. O teste demonstrou que para as variáveis: altura $(\mathrm{p}=0,838)$, DAP $(\mathrm{p}=0,461)$, área basal $(\mathrm{p}=0,713)$, número de indivíduos $(\mathrm{p}=0,653)$ e número de espécies $(\mathrm{p}=0,868)$ não houve diferenças estatisticamente significativas.

Palavras-chave: Florística; floresta tropical; unidade de conservação.
\end{abstract}

\begin{abstract}
Plot dimension comparison for vegetation analysis in an Atlantic Forest fragment, Aracaju, SE. In an Atlantic Forest fragment, located at the Environmental Protection Area of "Morro do Urubu", a vegetation analysis was conducted in order to compare different plot dimensions. The area was divided into 314 plots of $20 \times 100 \mathrm{~m}$, and then 15 sampling points were randomly selected, 15 plots of $20 \times 50 \mathrm{~m}\left(1000 \mathrm{~m}^{2}\right)$ and 15 plots of $10 \times 100 \mathrm{~m}\left(1000 \mathrm{~m}^{2}\right)$ were installed, from each one of the sampled points. In the plots, we measured the circumference at breast height (CBH - $1.30 \mathrm{~m}$ height) and the total height of individuals with $\mathrm{CBH}>31 \mathrm{~cm}$, using a measuring tape and a telescopic pole, respectively. In addition, we collected botanical material for scientific identification of species. The statistical test used to detect whether there was difference between independent treatments (different plot dimensions) was the Mann-Whitney test. The test showed that the following variables: height $(p=0$ 838), DAP $(p=0.461)$, basal area $(p=0.713)$, number of trees $(p=0.653)$ and number of species $(\mathrm{p}=0.868)$ were not statistically meaningful among them.

Keywords: Floristic; tropical forest; conservation units.
\end{abstract}

\section{INTRODUÇÃO}

Para uma melhor compreensão dos meios que proporcionam a recuperação de florestas, são importantes os estudos da composição, crescimento, estrutura e estoque florestal, que também auxiliam no planejamento de um manejo florestal sustentável, tornando possível o aproveitamento racional dos recursos florestais existentes. Ainda são necessários muitos estudos com a finalidade de perpetuar a atividade de manejo e mantê-la para gerações futuras, valendo ressaltar que o conhecimento desses

FLORESTA, Curitiba, PR, v. 45, n. 4, p. 735 - 744, out. / dez. 2015.

Nero, P. G. de O. et al.

ISSN eletrônico 1982-4688 / ISSN impresso 0015-3826 
parâmetros de crescimento, estrutura, estoque, etc., imprescindíveis em várias atividades, só é possível com a realização de levantamentos florestais (BONETES, 2003; AUGUSTYNCZIK et al., 2013).

O tamanho e forma das unidades amostrais têm implicações diretas sobre a definição da população estatística, a variância populacional (como consequência) e sobre os custos do levantamento. Também, o custo do levantamento e a precisão do mesmo são em função do tamanho da amostra, do tamanho das unidades amostrais e da distância entre as unidades amostrais (BATISTA, 2006). Em inventários florestais realizados na Mata Atlântica, o maior problema é a grande variabilidade de metodologias empregadas, principalmente quando se refere ao tamanho e forma de parcela, sendo que essa definição se torna um fator decisivo no processo de planejamento, antes da realização do inventário (OLIVEIRA, 2010; VIANNA et al., 2010).

A preocupação com as dimensões das unidades amostrais é um problema gerado no planejamento de um inventário florestal. Segundo Péllico Neto e Brena (1997), em geral, a forma e o tamanho das unidades amostrais têm sido decididas muito mais pela praticidade e operacionalidade de sua localização e demarcação em campo, do que por qualquer outra argumentação. Ao planejar experimentos, as características individuais de cada tipo de vegetação devem ser consideradas para a obtenção de resultados confiáveis. Uma das técnicas para minimizar o erro experimental e aumentar a precisão dos experimentos consiste na utilização de parcelas com forma e tamanho adequados (STORCK et al., 2006).

Atualmente há, na literatura, um déficit de trabalhos relacionados à comparação de dimensões de parcelas, principalmente em florestas de Mata Atlântica. A execução de trabalhos de pesquisa que determinem a melhor forma e tamanho de parcelas, nos diferentes biomas do país, é de extrema importância para auxiliar na obtenção de resultados satisfatórios. Sendo assim, visando acrescentar maior ciência ao assunto apresentado, o presente trabalho foi realizado com o objetivo de comparar duas dimensões de parcelas, para a realização da análise de vegetação, em uma área de Mata Atlântica localizada na APA Morro do Urubu.

\section{MATERIAL E MÉTODOS}

\section{Área de estudo}

A área estudada está situada na unidade de conservação APA Morro do Urubu, inserida no município de Aracaju, em Sergipe, com um perímetro de $8.135,25 \mathrm{~m}$, localizada entre as coordenadas $37^{\circ}$ 03' $27^{\prime}$ 'W e $10^{\circ} 53^{\prime} 01 S^{\prime}$ 'abrangendo uma área de 213,87 há, onde 25,7 há, foram utilizados para a realização do experimento (PINTO et al., 2008). Limita-se ao Norte com o rio do Sal, ao Leste com o rio Sergipe e ao Sul e Oeste com as áreas urbanas da zona Norte do município (SILVA, 2004). Trata-se de uma região onde a área de influência pertence ao domínio da Mata Atlântica e a vegetação caracteriza-se pela presença de fragmentos florestais em estágio secundário de regeneração natural (COOTRAM, 2004).

Segundo o AMBIENTEC (2004), a área de estudo apresenta um clima quente e úmido, com período chuvoso de março a agosto, com as temperaturas médias anuais oscilando entre $23,5^{\circ} \mathrm{C}$ a $28,8^{\circ} \mathrm{C}$ e apresentando uma variação pluviométrica mensal de $49,0 \mathrm{~mm}$ a $315,0 \mathrm{~mm}$ (de dezembro a maio). A APA apresenta-se com caracterização topográfica de um morro formado por encostas arredondadas, com níveis de altura que variam de 40 a 50 metros. A leste apresenta uma superfície mais alta que se eleva abruptamente do nível da Planície Litorânea, alcançando até 90 metros, onde se instala o Morro do Urubu (SILVA, 2004).

\section{Obtenção de dados}

Para a determinação da área de estudo foi obtido o mapa da APA através do Atlas Digital de Sergipe (2012), que possui toda a dimensão do morro do Urubu georreferenciada. Posteriormente, a área foi toda dividida em unidades amostrais de $20 \mathrm{~m}$ x $100 \mathrm{~m}$, que foram numeradas de forma ordenada. $\mathrm{O}$ processo de amostragem utilizado foi o Aleatório Simples e 15 unidades amostrais foram sorteadas. A partir do ponto inicial de cada unidade amostral sorteada foram instaladas duas parcelas, sendo uma de $20 \mathrm{~m} \times 50 \mathrm{~m}$ (tratamento 1) e outra de $10 \mathrm{~m} \times 100 \mathrm{~m}$ (tratamento 2), totalizando 30 parcelas de $1000 \mathrm{~m}^{2}$. Nessas parcelas foi realizada a medição de todos os indivíduos com CAP (Circunferência à Altura do Peito) $>31,4 \mathrm{~cm}(10 \mathrm{~cm}$ de DAP) (REDEMAP, 2007). Foram tomadas as medidas de CAP $(\mathrm{cm})$ e altura total $(\mathrm{m})$ desses indivíduos, utilizando fita métrica e vara telescópica, respectivamente. 
Após a medição das parcelas foi coletado o material botânico de cada espécie mensurada, sendo que o mesmo foi herborizado e a identificação dos exemplares foi realizada com o auxilio de chaves taxonômicas, consulta à especialistas, consulta à literatura especializada e através da comparação de exsicatas, valendo ressaltar que o sistema de classificação utilizado para a identificação das espécies foi o APG III (APG, 2009).

\section{Análise estatística}

As variáveis analisadas entre os tratamentos considerados foram: altura, DAP, área basal, número de indivíduos por parcela e número de espécies por parcela. Inicialmente, foram realizados testes para verificar a distribuição das variáveis utilizadas na comparação dos dois tamanhos de parcelas. Quando as variáveis possuíam valores acima de um (no caso do DAP, da altura total, do número de indivíduos e número de espécies) utilizou-se o teste de Shapiro-Wilks. Já para a variável com valores entre zero e um (área basal), o teste utilizado foi o de Lilliefors. Como algumas variáveis não apresentaram distribuição normal $(\mathrm{p}>0,05)$ optou-se pela realização de testes não paramétricos em todas as variáveis, sendo utilizado o teste de Mann-Whitney para detectar diferença significativa $(\mathrm{p}<0,05)$ entre tratamentos independentes. Todas as análises estatísticas foram realizadas utilizando-se o programa StatSoftStatistica versão 11, licenciado para Universidade Federal de Sergipe.

Além dos testes, foi também utilizado o Box-plot (gráfico de caixas) com a finalidade de possibilitar a melhor visualização do comportamento das variáveis entre os dois grupos de análise. Através desses gráficos, pode-se observar a distribuição dos tratamentos em relação à homogeneidade dos dados, valores de tendência central, valores máximos e mínimos.

Para comparação da eficiência das duas dimensões de parcelas utilizadas no presente estudo efetuou-se o cálculo da eficiência relativa (E), obtido através da fórmula proposta por Freese, em 1962, citada por Péllico Netto e Brena (1997):

$$
E=\frac{1}{T * C V^{2}}
$$

em que: $\mathrm{E}=$ eficiência relativa; $\mathrm{T}$ = tempo de medição; $\mathrm{CV}=$ coeficiente de variação.

\section{RESULTADOS E DISCUSSÃO}

De acordo com os testes Shapiro-Wilks e Lilliefors, se o valor calculado da estatística do teste for significativo $(\mathrm{p}<0,05)$ rejeita-se a hipótese de que a distribuição estudada é normal, ou seja, para a distribuição ser considerada normal o valor de $\mathrm{p}$ deve ser maior que 0,05 . Foi constatado que existe aderência a uma distribuição normal para todas as variáveis, em todos os tratamentos, exceto para a variável área basal e número de espécies, no tratamento dois, que apresentaram valor de $\mathrm{p}<0,05$ (Tabela 1).

Tabela 1. Testes de Lilliefors, Shapiro-Wilks para verificar a distribuição das variáveis: Altura total (m), DAP $(\mathrm{cm})$, Área basal $(\mathrm{cm})$, Número de indivíduos e Número de espécies.

Table 1. Lilliefors, Shapiro-Wilks test to verify the distribution of the variables: height, DAP, basal area, number of individuals and number of species.

\begin{tabular}{lcccc}
\hline \multirow{2}{*}{ Teste } & \multicolumn{2}{c}{ Tratamento 1 (20 x 50m) } & \multicolumn{2}{c}{ Tratamento 2 $(\mathbf{1 0}$ x 100 m) } \\
\cline { 2 - 5 } & Lilliefors & Shapiro-Wilks & Lilliefors & Shapiro-Wilks \\
\hline Altura $(\mathrm{m})$ & 0,20 & 0,041 & 0,200 & 0,420 \\
DAP $(\mathrm{cm})$ & $>0,20$ & 0,009 & 0,070 & $>0,200$ \\
Área Basal $(\mathrm{cm})$ & $<0,10$ & 0,002 & 0,010 & $<0,010$ \\
№ de indivíduos & 0,20 & 0,295 & $>0,200$ & 0,489 \\
№ de espécies & $>0,20$ & 0,493 & $<0,010$ & 0,005 \\
\hline
\end{tabular}

\section{Número de indivíduos por parcela}

Foram encontrados 851 indivíduos nas 30 unidades amostrais distribuídas Nas 15 parcelas de $20 \times 50 \mathrm{~m}$ foram medidas 438 árvores e nas outras 15 parcelas, de $10 \times 100 \mathrm{~m}, 413$ árvores. Pode-se verificar que nas parcelas de $20 \times 50 \mathrm{~m}$ o número de indivíduos medidos foi um pouco maior (25).

FLORESTA, Curitiba, PR, v. 45, n. 4, p. 735 - 744, out. / dez. 2015.

Nero, P. G. de O. et al.

ISSN eletrônico 1982-4688 / ISSN impresso 0015-3826

DOI: $10.5380 /$ rf.v45i4.37815 
Oliveira (2010), também utilizando parcelas de $10 \times 100 \mathrm{~m}$ e $20 \times 50 \mathrm{~m}$ em uma área de Floresta Amazônica, localizada na BR-174, entre as localidades de Manaus e Boa Vista, observou que as parcelas de dimensões diferentes apresentaram uma pequena diferença na quantidade de árvores mensuradas, sendo o tamanho de $20 \times 50 \mathrm{~m}$ o que apresentou o maior número de árvores por parcela. Porém essa diferença foi mínima, não sendo estatisticamente significativa.

Apesar das parcelas possuírem a mesma área, diferentes dimensões podem alterar a quantidade de árvores mensuradas ou abranger maior ou menor variabilidade da floresta. No presente estudo as parcelas pertencentes ao tratamento 1 apresentaram um maior número de indivíduos amostrados em relação as parcelas do tratamento 2. Esse resultado se torna contrário ao trabalho realizado por Chaves (1993), numa floresta de Mata Atlântica no interior de Minas Gerais, onde o autor verificou que parcelas em torno de 100 metros de comprimento são mais eficientes no método de amostragem ao realizar o inventário de acordo com os parâmetros fitossociológicos, do que parcelas de 50 metros de comprimento, independente do tamanho de sua largura, que pode variar entre 5 e $20 \mathrm{~m}$.

Considerando-se o tratamento 1 (parcelas de $20 \times 50 \mathrm{~m}$ ) verificou-se a ocorrência de 10 a 60 árvores nas parcelas, o que gera uma grande variabilidade no número de árvores desse tratamento. Já nas parcelas de $10 \times 100 \mathrm{~m}$ (tratamento 2) foi observado um número de 12 a 52 árvores por parcela, mostrando uma variabilidade menor quando comparado ao primeiro tratamento, como pode ser observado na figura 1 .

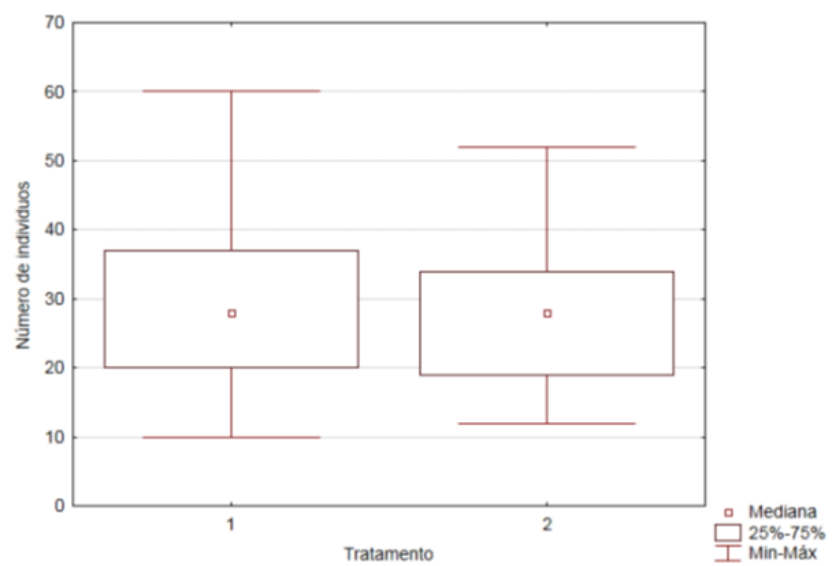

Figura 1. Variação do número de indivíduos considerando os diferentes tratamentos (T1 - 20 x $50 \mathrm{~m}$ e $\mathrm{T} 2-10 \times 100 \mathrm{~m})$.

Figure 1. Number of trees variation considering the different treatments (T1-20 x 50 m and T2 $10 \times 100 \mathrm{~m})$.

\section{Número de espécies por parcela}

No levantamento florístico realizado na área foram amostrados 851 indivíduos arbóreos representados por 50 espécies, distribuídas em 22 famílias. As famílias que apresentaram maior riqueza de espécies foram: Fabaceae (14), Myrtaceae (7) e Anacardiaceae (4). Vale ressaltar que as espécies Tapirira guianensis Aubl. (pau-pombo), Eschweilera ovata (Cambess.) Mart. Ex Miers (biriba), Lecythis sp. (sapucaia), Protium heptaplyllum (Aubl.) Marchand (amescla), Spondias mombin L.(cajá) e Cupania revoluta Radlk. (camboatã) foram as que tiveram maior predominância na APA.

Foi observado que as áreas amostradas apresentavam diferenças fisionômicas entre si, sendo algumas áreas consideradas mais desenvolvidas, pois apresentaram árvores mais altas e com maiores circunferências à altura do peito, e outras áreas menos desenvolvidas, pois apresentaram árvores com menores alturas e circunferências, o que também pôde ser observado no trabalho de Cardoso (2011). Segundo o autor, as diferenças fisionômicas encontradas na APA podem estar relacionadas ao relevo muito acidentado característico da região, além do fato de que determinadas áreas sofrem um severo processo erosivo causado pelo uso desordenado dos recursos naturais. Esse uso sem critérios pode ter ocasionado impactos ambientais negativos e localizados, provenientes de alterações no sistema ecológico, como disponibilidade de água e nutrientes. 
As parcelas do tratamento $2(10 \times 100 \mathrm{~m})$ foram as que apresentaram a maior quantidade de espécies (48), isto é, com relação a variável espécie, este tratamento abrangeu uma maior variabilidade. Já nas parcelas de tratamento $1(20 \mathrm{~m} \times 50 \mathrm{~m})$ foram identificadas 45 espécies, 3 a menos que no tratamento 2 .

Encinas et al. (2009), ao realizarem um trabalho sobre contribuição dendrométrica em levantamentos fitossociológicos, citam que o número de espécies é o atributo mais utilizado para descrever a taxocenose, uma vez que é uma expressão que permite fornecer uma informação segura de sua diversidade, sendo que o tamanho e a forma de parcelas são fatores determinantes para conseguir resultados mais satisfatórios. No presente estudo, foi constatado que as parcelas do tratamento $2(10 \times 100 \mathrm{~m})$ foram as que apresentaram uma maior variação no número de espécies (Figura 2), verificando-se de 5 a 15 espécies identificadas por unidade amostral. Nas parcelas de tamanho $20 \times 50 \mathrm{~m}$, a quantidade de espécies encontradas foi inferior, sendo encontradas de 4 a 11 espécies por unidade amostral.

Observa-se que as parcelas do tratamento $2(10 \times 100 \mathrm{~m})$ foram as que apresentaram resultados mais satisfatórios para estudos de levantamento florístico, pela maior quantidade de espécies identificadas, conseguindo captar melhor a diversidade que o bioma Mata Atlântica normalmente apresenta, além de ressaltar a importância que a área pode ter para a conservação da biodiversidade e preservação de espécies do bioma.

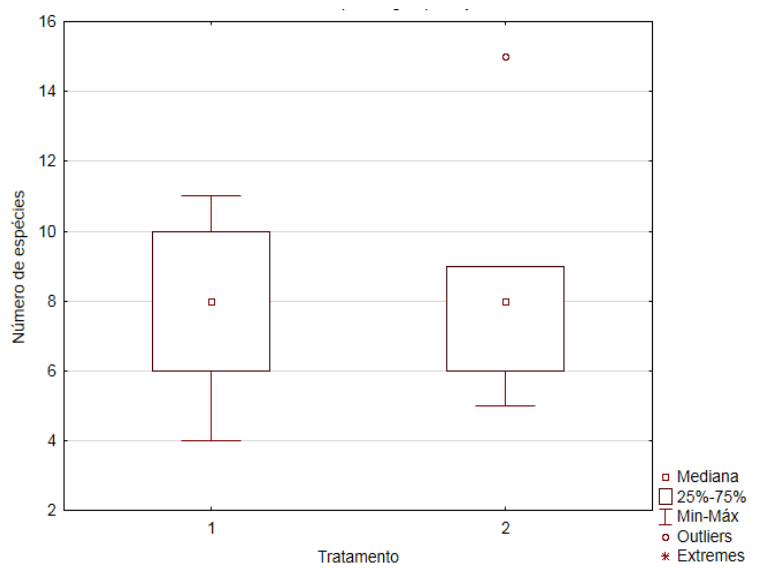

Figura 2. Variação do número de espécies considerando os diferentes tratamentos (T1 - 20 x 50 m e T2 $10 \times 100 \mathrm{~m})$.

Figure 2. Number of species variation considering the different treatments $(\mathrm{T} 1-20 \times 50 \mathrm{~m}$ and $\mathrm{T} 2-$ $10 \times 100 \mathrm{~m})$.

\begin{abstract}
Altura
O conhecimento das taxas de crescimento vertical da floresta é um dos parâmetros que regem a sua dinâmica, visto que as mudanças ocorridas em sua estrutura são de fundamental importância para se elaborar um plano de manejo. Os resultados das análises estruturais permitem fazer deduções sobre a origem, características ecológicas e sinecológicas, dinamismo e tendências do futuro desenvolvimento das florestas (CORAIOLA; PÉLLICO NETTO, 2003). No presente estudo, verificou-se uma altura média de 9,2 metros nas unidades amostrais de 20 x $50 \mathrm{~m}$ e de 9,1 metros nas unidades amostrais de $10 \mathrm{~m}$ x $100 \mathrm{~m}$. Pode-se observar que a diferença entre os tratamentos, considerando as médias das alturas, foi de apenas 0,10 metros.

De acordo com os dados coletados, observou-se que as parcelas do tratamento 1 ( $20 \times 50 \mathrm{~m})$ foram as que apresentaram árvores variando de 7,2 a 12,5 metros de altura (variação de 5,3 m). Já as parcelas do tamanho $10 \times 100 \mathrm{~m}$ apresentaram árvores de 6,8 a 12,2 metros (variação de 5,4 m), ou seja, o tratamento 2 abrangeu uma maior variação em relação à variável altura (Figura 3).
\end{abstract}

\title{
DAP e Área basal
}

Os diâmetros e circunferências são medidas fundamentais em estudos de dendrometria e servem de base para medições e estimações da área basal, volume, crescimento, classificação de local, comparação de variáveis, etc. (LEMOS, 2010). Nas parcelas de 20 x $50 \mathrm{~m}$ foi verificado um DAP médio de $15,8 \mathrm{~cm}$ e nas

FLORESTA, Curitiba, PR, v. 45, n. 4, p. 735 - 744, out. / dez. 2015.

Nero, P. G. de O. et al.

ISSN eletrônico 1982-4688 / ISSN impresso 0015-3826

DOI: $10.5380 /$ rf.v45i4.37815 
parcelas de $10 \times 100 \mathrm{~m}$ esse resultado foi de $15,7 \mathrm{~cm}$, onde pode ser percebida uma diferença de apenas $0,1 \mathrm{~cm}$ entre os tratamentos utilizados. Para a variável área basal foi observado uma diferença média de $0,000197 \mathrm{~m}^{2} \cdot \mathrm{ha}^{-1}$ entre os tratamentos, onde o tratamento $1(20 \times 50 \mathrm{~m})$ apresentou área basal média de $0,019942 \mathrm{~m}^{2}$.ha $\mathrm{a}^{-1}$ e o tratamento 2 (10 x $\left.100 \mathrm{~m}\right)$ área basal média de $0,020139 \mathrm{~m}^{2} \cdot \mathrm{ha}^{-1}$.

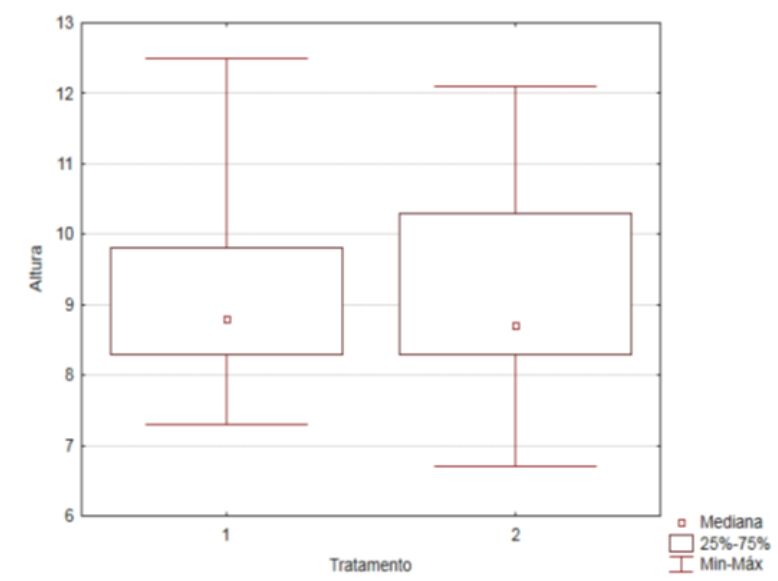

Figura 3. Variação da altura das árvores considerando os diferentes tratamentos (T1 - 20 x 50 m e T2 $10 \times 100 \mathrm{~m})$.

Figure 3. Variation of the height of species considering the different treatments (T1 - 20 x $50 \mathrm{~m}$ and T2 $10 \times 100 \mathrm{~m})$.

Em um trabalho realizado por Bonetes (2003) sobre o tamanho de parcelas e intensidade amostral para estimar o estoque e índices fitossociológicos de uma Floresta Ombrófila Mista, os valores estimados da área basal média por hectare, do erro real e do erro de amostragem, para as diferentes intensidades de amostragem e para os diferentes tamanhos de unidades amostrais, foram muito próximos entre as parcelas de $20 \times 50 \mathrm{~m}$ e $10 \times 100 \mathrm{~m}$. Neste caso, verificou-se que ambos os tamanhos apresentaram área basal de 30 a $35 \mathrm{~m}^{2} / \mathrm{ha}$, não havendo diferença significativa entre elas.

Augustynczik et al. (2013), ao realizarem um estudo comparando parcelas de tamanho 20 x $50 \mathrm{~m}$ e 10 x 100 m no remanescente de Floresta Ombrófila Mista no Paraná, avaliando o tamanho de parcelas e a intensidade de amostragem em inventários florestais, verificaram que as unidades amostrais não possuíam diferença significativa entre si, havendo uma diferença de $0,5 \%$ entre as parcelas, tanto no erro real como no erro de amostragem.

As parcelas do tratamento $2(10 \times 100 \mathrm{~m})$ foram as que apresentaram os maiores valores de DAP e área basal, sendo observada a ocorrência de árvores com $12,0 \mathrm{~cm}$ a 22,3 cm de DAP (variação de $10,3 \mathrm{~cm}$ ) e $0,011965 \mathrm{~m}^{2} \cdot h^{-1}$ a $0,029277 \mathrm{~m}^{2} \cdot \mathrm{ha}^{-1}$ de área basal (variação de $0,017312 \mathrm{~m}^{2} \cdot \mathrm{ha}^{-1}$ ). Nas unidades amostrais de $20 \times 50 \mathrm{~m}$, verificou-se que o DAP variou de $12,6 \mathrm{~cm}$ a $21,5 \mathrm{~cm}$ (variação de $8,9 \mathrm{~cm}$ ) e a área basal de $0,012407 \mathrm{~m}^{2} \cdot \mathrm{ha}^{-1}$ a $0,036208 \mathrm{~m}^{2} \cdot \mathrm{ha}^{-1}$ (variação de $0,023801 \mathrm{~m}^{2} \cdot$ ha ${ }^{-1}$ ), podendo ser percebido que o tratamento $2(10 \times 100 \mathrm{~m})$ abrangeu uma maior variação em relação à variável DAP (Figuras 4 e 5).

Além dos testes foi também utilizado o Box-plot (gráfico de caixas) com a finalidade de possibilitar uma melhor visualização do comportamento das variáveis entre os dois grupos de análise. Através desses gráficos, pôde-se observar a distribuição dos tratamentos em relação à homogeneidade dos dados, valores de tendência central e valores máximos e mínimos.

\section{Teste Mann-Whitney}

Para verificar se existe diferença estatística entre as variáveis nos tratamentos considerados foi realizado o teste de Mann-Whitney, que comprovou que as mesmas não foram significativas ( $p>0,05$ ), não havendo distinção entre elas quando consideramos as duas dimensões de parcelas utilizadas no presente estudo. 


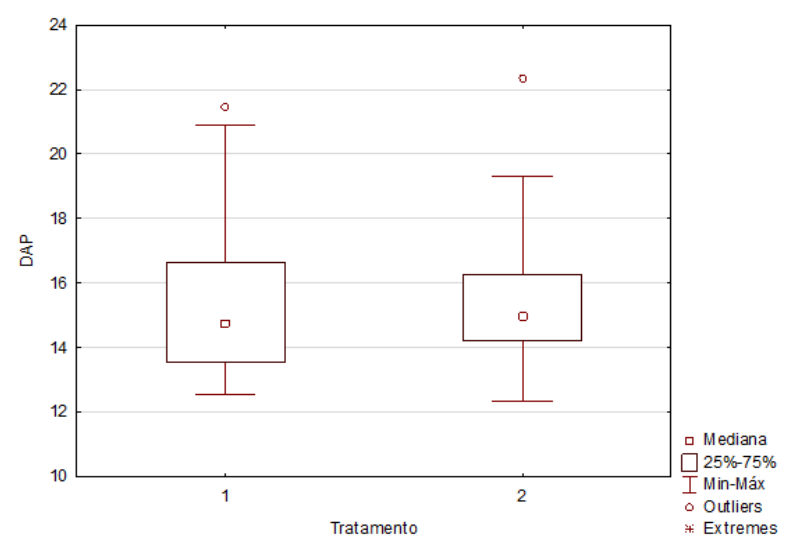

Figura 4. Variação do DAP das árvores considerando os diferentes tratamentos (T1 - 20 x $500 \mathrm{~m}$ e T2 $10 \times 100 \mathrm{~m}$ ).

Figure 4. Variation of trees DBH considering the different treatments $(\mathrm{T} 1-20 \times 50 \mathrm{~m}$ and $\mathrm{T} 2$ $10 \times 100 \mathrm{~m})$.

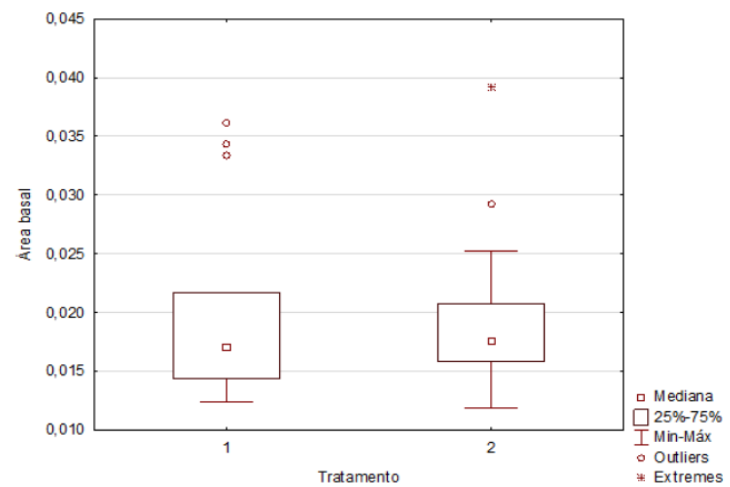

Figura 5. Variação da área basal considerando os diferentes tratamentos (T1-20x $50 \mathrm{~m}$ e T2 $10 \times 100 \mathrm{~m})$.

Figure 5. Variation of trees basal area considering the different treatments (T1 - 20 x $50 \mathrm{~m}$ and $\mathrm{T} 2$ $10 \times 100 \mathrm{~m})$.

Tabela 2. Resultado do teste Mann - Whitney.

Table 2. Result of the Mann - Whitney's test.

\begin{tabular}{lcccc}
\hline Mann -Whitney & Tratamento 1 & Tratamento 2 & Geral & P. Valor \\
\hline $\mathrm{N}^{\circ}$ de indivíduos & $29,2 \pm 11,869$ & $27,5 \pm 12,035$ & $28,4 \pm 11,775$ & 0,653 \\
$\mathrm{~N}^{\circ}$ de espécies & $7,8 \pm 2,242$ & $7,9 \pm 2,463$ & $7,9 \pm 2,315$ & 0,868 \\
Altura $(\mathrm{m})$ & $9,2 \pm 1,508$ & $9,1 \pm 1,649$ & $9,1 \pm 1,554$ & 0,838 \\
$\mathrm{DAP}(\mathrm{cm})$ & $15,6 \pm 2,891$ & $15,6 \pm 2,891$ & $15,7 \pm 2,571$ & 0,461 \\
Área Basal $(\mathrm{cm})$ & $0,0247 \pm 0,013$ & $0,0232 \pm 0,008$ & $0,0239 \pm 0,011$ & 0,713 \\
\hline
\end{tabular}

Eficiência relativa de acordo com o tempo de medição das variáveis e coleta dos dados

O tempo de medição de uma parcela é um dos fatores mais importantes na tomada de decisão de um trabalho. Por isso, é necessário escolher de forma racional qual tamanho e quais dimensões terá a unidade amostral a ser utilizada, com a necessidade de otimizar as atividades de campo, economizando tempo e recurso financeiro.

De acordo com o tempo de mensuração apresentado na tabela 3 , todas as variáveis $\left(\mathrm{n}^{\mathrm{o}}\right.$ de indivíduos, $\mathrm{n}^{\mathrm{o}}$ de espécies, altura, DAP e área basal) do tratamento 2 apresentaram eficiência relativa maior do que as variáveis do tratamento 1 . Vale ressaltar que a variável $\mathrm{n}^{-}$de indivíduos foi a que apresentou menor diferença entre a eficiência relativa dos dois tratamentos e, a área basal, a que apresentou maior diferença. Assim, podemos dizer que em áreas com características semelhantes às

FLORESTA, Curitiba, PR, v. 45, n. 4, p. 735 - 744, out. / dez. 2015.

Nero, P. G. de O. et al.

ISSN eletrônico 1982-4688 / ISSN impresso 0015-3826

DOI: $10.5380 /$ rf.v45i4.37815 
apresentadas nesse estudo, seria mais eficiente realizar inventários florestais utilizando-se parcelas com dimensão $10 \times 100 \mathrm{~m}$, já que esse tratamento mostrou maior eficiência relativa para todas as variáveis consideradas.

De acordo com o tempo de coleta (Tabela 3) o $\mathrm{n}^{\underline{0}}$ de indivíduos e $\mathrm{n}^{\mathrm{o}}$ de espécies mostraram a mesma eficiência relativa em ambos os tratamentos. Já a variável altura e DAP apresentaram uma pequena diferença $(0,005$ e 0,006 , respectivamente) e a variável área basal mostrou uma maior alternância nos resultados $(0,018)$, se comparado com as outras variáveis.

Tabela 3. Eficiência relativa do tempo médio de medição e coleta em cada tratamento analisado.

Table 3. Relative efficiency of measurement and collect time of each analyzed plot.

\begin{tabular}{lccccc}
\hline \multirow{2}{*}{ Variáveis } & & \multicolumn{2}{c}{ Medição } & \multicolumn{2}{c}{ Coleta } \\
& & T 1 & T 2 & T1 & T 2 \\
\hline \multirow{2}{*}{ Tempo (min) } & Média & 127 & 104 & 66 & 62 \\
& & & & & \\
\multirow{2}{*}{$\mathbf{N}^{\circ}$ de indivíduos } & CV\% & 0,410 & 0,440 & 0,410 & 0,440 \\
& ER & 0,019 & 0,022 & 0,037 & 0,037 \\
\multirow{2}{*}{$\mathbf{N}^{\circ}$ de espécies } & CV\% & 0,290 & 0,310 & 0,290 & 0,310 \\
& ER & 0,027 & 0,031 & 0,052 & 0,052 \\
\multirow{2}{*}{ Altura } & CV\% & 0,160 & 0,180 & 0,160 & 0,180 \\
& ER & 0,049 & 0,053 & 0,095 & 0,090 \\
\multirow{2}{*}{ DAP (cm) } & CV\% & 0,180 & 0,180 & 0,180 & 0,180 \\
& ER & 0,044 & 0,053 & 0,084 & 0,090 \\
\multirow{2}{*}{ Área Basal } & CV\% & 0,530 & 0,340 & 0,530 & 0,340 \\
& ER & 0,015 & 0,028 & 0,029 & 0,047 \\
\hline
\end{tabular}

\section{CONCLUSÕES}

- As parcelas do tratamento $2(10 \times 100 \mathrm{~m})$ detectaram maior variação em relação as variáveis: espécies por parcela, DAP, bem como consumiram menor tempo na mensuração das árvores.

- As parcelas do tratamento $1(20 \times 50 \mathrm{~m})$ abrangeram maior quantidade de árvores por parcela e as que detectaram maior variação de altura.

- De acordo com o tempo de medição das variáveis DAP, altura e coleta de exsicatas, o tratamento 2 foi o mais viável, por consumir menor tempo.

- Verificou-se, através do teste de Mann-Whitney, que nenhuma das variáveis testadas (número de indivíduos, número de espécies, altura, DAP e área basal) apresentou diferenças estatisticamente significativas entre os tratamentos comparados.

\section{REFERÊNCIAS}

AUGUSTYNCZIK, A. L. D.; MACHAdO, S. A.; FIGUEIRO FILHO, A.; PÉLliCO NETTO, S. Avaliação do tamanho de parcelas e de intensidade de amostragem em inventários florestais. Scientia Forestalis, Piracicaba, v. 41, n. 99, p. 361 - 368, 2013.

AMBIENTEC. Consultoria Ltda. Estudo de Impacto Ambiental e Relatório de Impacto Ambiental da Ponte sobre o Rio Sergipe. Aracaju, 2004.78 p.

ANGIOSPERM PHYLOGENY GROUP (APG) III. An update of the Angiosperm Phylogeny Group classification for the orders and families of flowering plants: APG III. Botanical Journal of the Linnean Society, v. 161, p. $105-121,2009$. 
BONETES, L. Tamanho de parcelas e intensidade amostral para estimar o estoque e índices fitossociológicos em uma Floresta Ombrófila Mista. 126 p. Dissertação (Mestrado em Ciências Florestais) - UFPA, Curitiba, 2003.

CARDOSO, D. S. Análise fitossociológica do remanescente de Mata Atlântica da Área de Proteção Ambiental Morro do Urubu, Aracaju - SE. 43 p. Monografia (Graduação em Biologia) - UFS, São Cristovão, 2011.

CHAVES, A. L. Determinação de tamanho e forma ideal de parcelas para fitossociologia e cálculo de volume de uma Mata Estacional Semidecídua Montana na microregião de Lavras - MG. 39 p. Monografia (Graduação em Engenharia Florestal) - UFLA, Lavras, 1993.

COOPERATIVA DOS TRABALHADORES AMBIENTALISTAS DE ALAGOAS (COOTRAM). Diagnóstico Ambiental da APA do Morro do Urubu. Aracaju, 2004. 62 p.

CORAIOLA, M.; PÉLLICO NETTO, S. Análise da estrutura horizontal de uma floresta estacional semidecidual localizada no município de Cássia-MG. Ciências Agrárias e Ambientais, Curitiba, v. 1, n. 2, p. 11 - 19, 2003.

ENCINAS, J. I.; REZENDE, A. V.; IMAÑA, C. R.; SANTANA, O. A. A Contribuição dendrométrica nos levantamentos fitossociológicos. DF, Brasília: Universidade de Brasília, 2009, 52 p.

LEMOS, S. Mensuração de déficit isoperimétrico na estimação da área basal em clones do híbrido Eucalyptus grandis $\mathbf{x}$ Eucalyptus urophylla. 31 p. Monografia (Graduação em Engenharia Florestal) UFES, Jerônimo Monteiro, 2010.

OLIVEIRA, M. M. Tamanho e forma de parcelas para inventários florestais de volume de madeira e estoque de carbono de espécies arbóreas na Amazônia central. 73 p. Dissertação (Ciências de Florestas Tropicais) - Instituto Nacional de Pesquisas da Amazônia, Manaus, 2010.

PÉLLICO NETTO, S.; BRENA, D. A. Inventário Florestal, Curitiba/PR, 1997. 316 p.

PINTO, J. B.; ANDRADE, J. R. L.; SILVA, C. E. Possibilidades de desenvolvimento do Ecoturismo na Área de Proteção Ambiental Morro do Urubu, Aracaju, SE. Revista Brasileira de Ecoturismo, São Paulo, v. 1, n. 1, p. 42 - 61. 2008.

REDE DE PARCELAS PERMANENTES DOS BIOMAS MATA ATLÂNTICA E PAMPA (REDEMAP). Manual de instalação e Medição de Parcelas Permanentes dos Biomas Mata Atlântica e Pampa. Curitiba: Funpar- Fundação Universidade Federal do Paraná, 2007. 40 p.

SERGIPE. Secretaria de Estado do Meio Ambiente e dos Recursos Hídricos. Atlas digital sobre recursos hídricos. Aracajú: SEMARH, 2012. 1 DVD.

SILVA, K. C. T. O urbano, o rural e o ambiental nas transformações do Bairro Porto Dantas, no norte da cidade de Aracaju-SE. 134 p. Dissertação (Mestrado em Geografia) - UFS, São Cristóvão, 2004.

STORCK, L.; BISOGNIN, D. A.; OLIVEIRA, S. J. R. Dimensões dos ensaios e estimativas do tamanho ótimo de parcela em batata. Pesquisa Agropecuária Brasileira. Brasília, v. 41, n. 6, p. 903 - 909, 2006.

VIANNA, A. L. M.; VIDAL, E. J.; GORENSTEIN, M. R.; BATISTA, J. L. F. Tamanho ótimo de parcela para estimativa de estoque de carbono da parte aérea lenhosa de fitofisionomias florestais na Amazônia oriental. Revista Floresta, Curitiba, v. 40, n. 2, p. 447 - 456, 2010. 